\title{
Urea Ammonium Nitrate as the Carrier for Herbicides in Winter Wheat
}

\author{
Nader Soltani*, Christy Shropshire, Peter H. Sikkema
}

Ridgetown Campus, University of Guelph, Ridgetown, Canada.

Email: "nsoltani@ridgetownc.uoguelph.ca

Received November $7^{\text {th }}, 2011$; revised December $18^{\text {th }}, 2011$; accepted January $14^{\text {th }}, 2012$

\begin{abstract}
Urea ammonium nitrogen can be used as a carrier for herbicides to provide growers with an option for the control of broadleaf weeds and spraying nitrogen fertilizer in one pass in winter wheat. Field studies (six in total) were seeded in the autumn of 2005, 2006 and 2007 at Exeter and Ridgetown, Ontario, Canada to determine if UAN can be used as a carrier for bromoxynil/MCPA, dichlorprop/2,4-D, dicamba/MCPA/mecoprop, or thifensulfuron/tribenuron applied postemergence (POST) at three application timings (approximately April 20, May 1 and May 10) in winter wheat. Winter wheat injury was as much as $4 \%, 5 \%, 4 \%$ and $5 \%$ for bromoxynil/MCPA, dichloroprop/2,4-D, dicamba/MCPA/ mecoprop or thifensulfuron/tribenuron, respectively. There was minimal visible winter wheat injury with treatments evaluated at 4 and 9 week after treatment. There was no significant reduction in winter wheat height or yield with herbicides evaluated at various application timings except for dicamba/MCPA/mecoprop treatment which reduced height $3 \%$ and yield $25 \%$ at May 10 compared with April 20 application timing. Herbicides carrier had no effect on winter wheat height or yield with evaluated herbicides. Based on this research there is potential for co-application of UAN and bromoxynil/MCPA, dichlorprop/2,4-D, dicamba/MCPA/mecoprop, or thifensulfuron/tribenuron applied (POST) early in the spring in winter wheat.
\end{abstract}

Keywords: Injury; Herbicide Sensitivity; Plant Height; Yield

\section{Introduction}

Winter wheat (Triticum aestivum L.) is important to the agriculture in Ontario where it ranks as the fourth largest field crop grown in the province after maize (Zea mays L.), soybean (Glycine max L.) and alfalfa (Medicago sativa L.) [1]. In 2010, winter wheat growers planted nearly 443,000 hectares and produced 2,207,000 MT of winter wheat with a farm gate value of more than $\$ 300,000,000$ [2]. Ontario growers like to include winter wheat in their crop rotation as this crop is seeded in narrow rows in the autumn allowing it to suppress weed growth. In addition the preplant and postharvest herbicide application timings allow for control of biennial and perennial weeds. The fibrous roots system of winter wheat can also improve soil structure [3]. Winter cereals also play an important role in the protection of light soils against water and wind erosion as their establishment in the autumn helps to anchor the soil during the winter and spring seasons [3]. Winter wheat can provide a 20 to $30 \%$ yield advantage over the spring wheat if it overwinters successfully [4]. Intensive agronomic practices, in-

"Corresponding author. cluding effective weed control and nitrogen fertilizer management, are needed for profitable production of this important field crop.

Growers often use bromoxynil/MCPA, dichlorprop/ 2,4-D, dicamba/MCPA/mecoprop, or thifensulfuron/tribenuron applied postemergence (POST) for the control annual, biennial and perennial broadleaf weeds in winter wheat. Nitrogen fertilizer such as $28 \%$ liquid urea-ammonium nitrate solution (UAN) is often used in winter wheat [5]. Application timing of winter wheat herbicides and topdress liquid UAN fertilizers may coincide. Coapplication of bromoxynil/MCPA, dichlorprop/2,4-D, dicamba/MCPA/mecoprop, and thifensulfuron/tribenuron with a liquid fertilizer such as UAN would allow growers to reduce the number of passes through the field thereby reducing fuel and labor costs, machinery depreciation, soil compaction, as well as mechanical damage to crop foliage [6-11].

UAN has been shown to increase winter wheat injury when used as the carrier for herbicides under some environments [12-18]. There are no published data on the effects of co-application of bromoxynil/MCPA, dichlorprop/2,4-D, dicamba/MCPA/mecoprop or thifensulfuron/ 
tribenuron applied POST with UAN in winter wheat under Ontario environmental conditions. In addition, information on the compatibility of UAN with bromoxynil/MCPA, dichlorprop/2,4-D, dicamba/MCPA/mecoprop, or thifensulfuron/tribenuron is very important to winter wheat growers since incompatibility in the tank can result in significant winter wheat crop injury and equipment damage as well as reduction in weed control. If tolerance is adequate, use of UAN as a carrier for bromoxynil/MCPA, dichlorprop/2,4-D, dicamba/MCPA/ mecoprop, or thifensulfuron/tribenuron would provide growers with an option for the control of broadleaf weeds and spraying nitrogen fertilizer in one pass in winter wheat. Also, determining the appropriate application timing of UAN is critical as improper application timing of UAN can result in leaf burn and other foliar injury in winter wheat [16].

The objective of this research was to determine if the use of UAN as a carrier for bromoxynil/MCPA, dichlorprop/2,4-D, dicamba/MCPA/mecoprop or thifensulfuron/ tribenuron applied postemergence would result in increased injury, plant height reduction or yield reduction at various application timings in winter wheat.

\section{Materials and Methods}

Field studies were seeded in the autumn of 2005, 2006 and 2007 at the Huron Research Station, Exeter, Ontario, Canada and at the University of Guelph, Ridgetown Campus, Ridgetown, Ontario, Canada (total of six studies). The soil at Exeter was a Brookston clay loam (Orthic Humic Gleysol) with $33 \%$ sand, $35 \%$ silt, $32 \%$ clay, $3.4 \%$ organic matter and $\mathrm{pH}$ of 8.0 in $2005,28 \%$ sand, $38 \%$ silt, $34 \%$ clay, $4.1 \%$ organic matter and $\mathrm{pH}$ of 7.9 in 2006 , and $31 \%$ sand, $38 \%$ silt, $31 \%$ clay, $4.4 \%$ organic matter and $\mathrm{pH}$ of 7.9 in 2007 . The soil at Ridgetown was a Watford (Grey to Brown Brunisolic)-Brady (Gleyed Brunisolic Grey to Brown Luvisol, mixed) sandy loam with $52 \%$ sand, $28 \%$ silt, $20 \%$ clay, $5.9 \%$ organic matter and $\mathrm{pH}$ of 7.2 in $2005,45 \%$ sand, $29 \%$ silt, $26 \%$ clay, $5.2 \%$ organic matter and $\mathrm{pH}$ of 6.6 in 2006 , and $30 \%$ sand, $36 \%$ silt, $33 \%$ clay, $5.6 \%$ organic matter and $\mathrm{pH}$ of 7.4 in 2007 .

The study was established as a three-way factorial in a randomized complete block design with four replications. Factor one was herbicide treatment (bromoxynil/MCPA, dichlorprop/2,4-D, dicamba/MCPA/mecoprop, or thifensulfuron/tribenuron), factor 2 was carrier solution (water or $28 \%$ UAN at $200 \mathrm{l} \cdot \mathrm{ha}^{-1}$ ) and factor 3 was application timing (approximately April 20, May 1 and May 10 as POST 1, 2 and 3, respectively). Treatments included a non-treated check. Plots were $2 \mathrm{~m}$ wide by $10 \mathrm{~m}$ long at Exeter and $2 \mathrm{~m}$ wide by $8 \mathrm{~m}$ long at Ridgetown. Winter wheat "Pioneeer 25R47" was seeded with a double disc drill at $150 \mathrm{~kg} \cdot \mathrm{ha}^{-1}$ in rows spaced $17.5 \mathrm{~cm}$ apart at a depth of $4 \mathrm{~cm}$ from mid-October to early November.

Herbicides were applied on approximately April 20, May 1 and May 10 of each year for POST 1, POST 2 and POST 3 application timing, respectively. Treatments were applied with a $\mathrm{CO}_{2}$ pressurized backpack sprayer calibrated to deliver $200 \mathrm{~L} \cdot \mathrm{ha}^{-1}$ aqueous solution at $241 \mathrm{kPa}$. The boom was $1.5 \mathrm{~m}$ long with four Hypro ULD 120-02 nozzle tips (Hypro, New Brighton, MN, USA) spaced 50 $\mathrm{cm}$ apart. All plots including the untreated control were kept weed-free as needed. Additional UAN was applied 3 - 4 days after herbicide application to those plots where UAN was not used as the herbicide carrier so that each plot received an equivalent amount of nitrogen.

Crop injury was rated visually on a scale of 0 to $100 \%$ $(0=$ no visible injury, and $100=$ plant death $)$ at $1,2,4,6$ and 9 weeks after treatment (WAT). Ten plants were randomly selected per plot and the height from the soil surface to the highest growing point of each plant was measured at 6 WAT. Winter wheat was harvested in late July using a plot combine and yields were adjusted to $14.5 \%$ moisture.

All data were subjected to analysis of variance. Tests were combined over environments and analyzed using the MIXED procedure of SAS (The SAS System for Windows, Release 9.2., Cary, NC, USA). Variances were partitioned into the random effects of environment (comprising years and locations), blocks within environment, and the interactions with fixed effects (herbicide treatment, carrier solutions and application timings). Significance of random effects were tested using a Z-test of the variance estimate and fixed effects were tested using F-tests. Error assumptions of the variance analyses (random, homogeneous, normal distribution of error) were confirmed using residual plots and the ShapiroWilk normality test. To meet the assumptions of normality, injury at 1, 2 and 6 WAT were square-root transformed and injury at 4 (Exeter $2006 \&$ 2007) and 9 WAT were arcsine square-root transformed. Means were converted back to the original scale for presentation of results. Means were separated using Fisher's protected LSD at $\mathrm{P}=0.05$.

\section{Results and Discussion}

Injury data 4 weeks after treatment (WAT) at Ridgetown (all years), 6 WAT at Ridgetown (all years) and Exeter 2006 and 9 WAT (2006 \& 2007) at both Ridgetown and Exeter were all zero and could not be combined with the other environments (the zero data are not presented). Data had to be separated for injury 1 WAT $(2005,2006$ \& 2007), 4 WAT (Exeter 2005, Exeter 2006 \& 2007), and yield (2005, $2006 \& 2007)$. Herbicide treatment was significant for injury 4 WAT (Exeter 2005 only), herbi- 
cide carrier was significant for injury 1 WAT (2006 \& 2007 only) and injury 2 WAT, and application timing was significant for injury 2 WAT and injury 4 WAT (Exeter 2005 only). Seed moisture content was not significant for herbicides evaluated at various application timings and with water or UAN herbicide carrier (not shown).

\subsection{Injury}

Analysis of the main effects indicated that in 2005 at 4
WAT, dicamba/MCPA/mecoprop and thifensulfuron/ tribenuron caused greater injury than bromoxynil/MCPA and dichloroprop/2,4-D when data were combined over herbicide carriers and application timings (Table 1). Also, when there was any difference in application timing, the later application timing (May 10 $0^{\text {th }}$ ) caused greater injury than earlier application timings (April $20^{\text {th }}$ or May $1^{\text {st }}$ ) (Table 1).

Winter wheat injury 1 WAT (2006 and 2007) ranged from $0.4 \%$ to $3.6 \%, 0.4 \%$ to $5.1 \%, 3.2 \%$ to $3.9 \%$ and

Table 1. Significance of main effects and interactions for percent visual injury, height and yield of winter wheat. Means were transformed back to original scale for presentation. Means followed by a different letter within a column are significantly different according to Fisher's Protected LSD at $\mathbf{P}<\mathbf{0 . 0 5}$. Means for a main effect were separated only if there were no significant interactions involving that main effect. ${ }^{\text {a }}$

\begin{tabular}{|c|c|c|c|c|c|c|c|c|c|c|}
\hline \multirow{3}{*}{ Main effects ${ }^{b}$} & \multicolumn{7}{|c|}{ Winter wheat injury } & & & \\
\hline & \multicolumn{2}{|c|}{1 WAT } & \multirow{2}{*}{2 WAT } & \multicolumn{2}{|c|}{4 WAT } & \multirow{2}{*}{6 WAT } & \multirow{2}{*}{9 WAT } & \multirow{2}{*}{ Height } & \multicolumn{2}{|r|}{ Yield } \\
\hline & 2005 & $2006 \& 2007$ & & 2005 & $2006 \& 2007$ & & & & 2005 & $2006 \& 2007$ \\
\hline & \multicolumn{5}{|c|}{$\%$} & \multirow{3}{*}{ NS } & \multirow{3}{*}{ NS } & $\mathrm{cm}$ & \multicolumn{2}{|r|}{ MT $\cdot \mathrm{ha}^{-1}$} \\
\hline Herbicide treatment & NS & NS & NS & $* *$ & NS & & & NS & NS & NS \\
\hline Untreated & & & & & & & & 74 & 5.5 & 6.4 \\
\hline Bromoxynil/MCPA & 0.5 & 2.0 & 0.8 & 0.1 & 0 & 0 & 0 & 74 & 5.4 & 6.4 \\
\hline Dichlorprop/2,4-D & 0.9 & 2.7 & 1.1 & 0.7 & 0 & 0.4 & 0.1 & 74 & 5.5 & 6.5 \\
\hline Dicamba/MCPA/mecoprop & 1.5 & 3.6 & 1.3 & 2.5 & 0 & 1.9 & 1.5 & 74 & 5.3 & 6.4 \\
\hline Thifensulfuron/tribenuron $^{c}$ & 1.2 & 3.7 & 1.2 & 2.0 & 0.1 & 1.1 & 0.1 & 74 & 5.7 & 6.4 \\
\hline SE & 0.4 & 0.4 & 0.2 & 0.2 & 0 & 0.5 & 0.4 & 1 & 0.1 & 0.1 \\
\hline Application timing & NS & NS & $*$ & $* *$ & NS & NS & NS & NS & NS & NS \\
\hline April 20 & 0.2 & 1.2 & 0.7 & 0.4 & 0 & 0.9 & 0 & 74 & 5.6 & 6.6 \\
\hline May 1 & 0.4 & 3.8 & 0.7 & 0.6 & 0 & 0.2 & 0.1 & 74 & 5.6 & 6.4 \\
\hline May 10 & 3.1 & 4.4 & 2.1 & 3.0 & 0.1 & 1.2 & 1.1 & 74 & 5.2 & 6.3 \\
\hline SE & 0.2 & 0.3 & 0.1 & 0.2 & 0 & 0.4 & 0.3 & 1 & 0.1 & 0.1 \\
\hline$U A N^{d}$ & NS & $* *$ & $* *$ & NS & NS & NS & NS & NS & NS & NS \\
\hline Water carrier & 0.4 & $1.5 \mathrm{a}$ & 0.5 & 1.3 & 0 & 0.6 & 0.2 & 74 & 5.5 & 6.5 \\
\hline UAN carrier & 1.8 & $4.8 \mathrm{~b}$ & 1.8 & 1.4 & 0.1 & 0.8 & 0.3 & 74 & 5.5 & 6.4 \\
\hline $\mathrm{SE}$ & 0.2 & 0.2 & 0.1 & 0.2 & 0 & 0.4 & 0.4 & 1 & 0.1 & 0.1 \\
\hline Interactions & & & & & & & & & & \\
\hline $\mathrm{H} \times \mathrm{T}$ & NS & $* *$ & NS & $* *$ & $*$ & NS & $*$ & $*$ & $* *$ & NS \\
\hline $\mathrm{H} \times \mathrm{U}$ & NS & NS & NS & $* *$ & NS & NS & NS & NS & NS & NS \\
\hline $\mathrm{T} \times \mathrm{U}$ & $* *$ & NS & $* *$ & NS & NS & NS & NS & NS & NS & $*$ \\
\hline $\mathrm{H} \times \mathrm{T} \times \mathrm{U}$ & $*$ & NS & NS & NS & $*$ & NS & NS & NS & NS & NS \\
\hline
\end{tabular}

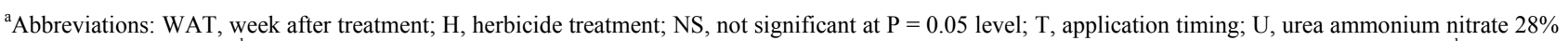
(UAN) solution carrier; ${ }^{\mathrm{b}}$ Significance at $\mathrm{P}<0.05$ and $\mathrm{P}<0.01$ levels denoted by $*$ and $* *$, respectively. ${ }^{\mathrm{c}}$ Included non-ionic surfactant at $0.2 \% \mathrm{v} / \mathrm{v}$. ${ }^{\mathrm{d}} \mathrm{Water}$ and UAN were applied at $2001 \cdot$ ha $^{-1}$. 
$1.5 \%$ to $5.2 \%$ for bromoxynil/MCPA, dichloroprop/2,4-D, dicamba/MCPA/mecoprop and thifensulfuron/tribenuron, respectively (Table 2). Generally winter wheat injury increased as the application timing was delayed although results were not always statistically significant. There was minimal visible injury (1.2\% or less) at 4 and 9
WAT when the herbicides were applied at April 20 or May 1. At 4 WAT (2005) dicamba/MCPA/mecoprop and thifensulfuron/tribenuron caused $7.6 \%$ and $4.2 \%$ injury, respectively (Table 2). At 9 WAT (in 2005), injury was only significant for dicamba/MCPA/mecoprop treatment applied at May 10 application timing (Table 2).

Table 2. Percent visual injury, height and yield of winter wheat for four herbicide treatments as a function of application timing. Means followed by the same letter within a column (a-b) or row (X-Z) for each section are not significantly different according to Fisher's Protected LSD at $\mathbf{P}<0.05$. $^{\text {a }}$

\begin{tabular}{|c|c|c|c|c|c|}
\hline \multirow{2}{*}{$\begin{array}{l}\text { Application timing } \\
\text { by Variable }\end{array}$} & \multicolumn{5}{|c|}{ Winter wheat injury } \\
\hline & Untreated & Bromoxynil/MCPA & Dichlorprop/2,4-D & Dicamba/MCPA/mecoprop & Thifensulfuron/tribenuron ${ }^{\mathrm{b}}$ \\
\hline & \multicolumn{5}{|c|}{$\%$} \\
\hline \multicolumn{6}{|l|}{$\begin{array}{l}\text { Injury } 1 \text { WAT } \\
(2006 \text { \& 2007) }\end{array}$} \\
\hline April 20 & $0 \mathrm{a} Z$ & $0.4 \mathrm{a} \mathrm{Y}$ & $0.4 \mathrm{a} Y$ & $3.2 \mathrm{a} \mathrm{X}$ & $1.5 \mathrm{a} \mathrm{Y}$ \\
\hline May 1 & 0 a $Z$ & $2.8 \mathrm{~b} \mathrm{Y}$ & $3.6 \mathrm{~b}$ YX & 3.9 a YX & $5.2 \mathrm{~b} \mathrm{X}$ \\
\hline May 10 & 0 a $Z$ & $3.6 \mathrm{~b} \mathrm{Y}$ & $5.1 \mathrm{~b} \mathrm{Y}$ & $3.7 \mathrm{a} \mathrm{Y}$ & $5.2 \mathrm{~b} \mathrm{Y}$ \\
\hline \multicolumn{6}{|l|}{$\begin{array}{c}\text { Injury } 4 \text { WAT } \\
\text { (2005) }\end{array}$} \\
\hline April 20 & 0 a $Z$ & 0 a ZY & $1.0 \mathrm{ab} \mathrm{Y}$ & 0 a ZY & 0.5 a ZY \\
\hline May 1 & $0 \mathrm{a} Z$ & 0 a ZY & $1.1 \mathrm{~b} \mathrm{Y}$ & 0 a ZY & $1.2 \mathrm{ab} \mathrm{Y}$ \\
\hline May 10 & $0 \mathrm{a} Z$ & $0.2 \mathrm{a} \mathrm{Z}$ & $0 \mathrm{a} Z$ & $7.6 \mathrm{~b} \mathrm{X}$ & $4.2 \mathrm{~b} \mathrm{Y}$ \\
\hline \multicolumn{6}{|l|}{$\begin{array}{l}\text { Injury } 4 \text { WAT } \\
\text { (2006 \& 2007) }\end{array}$} \\
\hline April 20 & $0 \mathrm{a} Z$ & $0 \mathrm{aZ}$ & $0 \mathrm{a} Z$ & $0 \mathrm{a} Z$ & $0 \mathrm{a} Z$ \\
\hline May 1 & $0 \mathrm{a} Z$ & $0 \mathrm{a} Z$ & $0 \mathrm{a} Z$ & $0 \mathrm{a} Z$ & 0 a Z \\
\hline May 10 & $0 \mathrm{a} Z$ & $0 \mathrm{a} Z$ & $0 \mathrm{a} Z$ & $0 \mathrm{a} Z$ & $0.7 \mathrm{~b} \mathrm{Y}$ \\
\hline \multicolumn{6}{|l|}{$\begin{array}{c}\text { Injury } 9 \text { WAT } \\
\text { (2005) }\end{array}$} \\
\hline April 20 & $0 \mathrm{a} Z$ & $0 \mathrm{aZ}$ & $0.1 \mathrm{a} \mathrm{Z}$ & $0 \mathrm{a} Z$ & $0 \mathrm{a} Z$ \\
\hline May 1 & $0 \mathrm{a} Z$ & $0.1 \mathrm{a} \mathrm{Z}$ & $0.2 \mathrm{a} \mathrm{Z}$ & $0.1 \mathrm{a} \mathrm{Z}$ & $0.1 \mathrm{a} \mathrm{Z}$ \\
\hline May 10 & $0 \mathrm{a} Z$ & $0 \mathrm{a} Z$ & $0.1 \mathrm{a} \mathrm{Z}$ & $9.7 \mathrm{~b} \mathrm{Y}$ & $0.4 \mathrm{a} \mathrm{Z}$ \\
\hline Height & \multicolumn{5}{|c|}{$\mathrm{cm}$} \\
\hline April 20 & 74 a Z & 74 a Z & 74 a Z & 75 a Z & 74 a Z \\
\hline May 1 & 74 a Z & 73 a Z & 74 a Z & 75 a Z & 74 a Z \\
\hline May 10 & 74 a ZY & 74 a ZY & 75 a Z & $73 \mathrm{~b} \mathrm{Y}$ & 74 a ZY \\
\hline Yield (2005) & \multicolumn{5}{|c|}{ MT $\cdot \mathrm{ha}^{-1}$} \\
\hline April 20 & $5.5 \mathrm{a} \mathrm{Z}$ & $5.5 \mathrm{a} \mathrm{Z}$ & $5.5 \mathrm{a} \mathrm{Z}$ & $5.7 \mathrm{a} \mathrm{Z}$ & $5.8 \mathrm{a} \mathrm{Z}$ \\
\hline May 1 & $5.5 \mathrm{a} \mathrm{Z}$ & $5.2 \mathrm{a} \mathrm{Z}$ & $5.6 \mathrm{a} \mathrm{Z}$ & $5.5 \mathrm{a} \mathrm{Z}$ & $5.8 \mathrm{a} \mathrm{Z}$ \\
\hline May 10 & $5.5 \mathrm{a} \mathrm{Z}$ & $5.4 \mathrm{a} \mathrm{Z}$ & $5.4 \mathrm{a} \mathrm{Z}$ & $4.3 \mathrm{~b} \mathrm{Y}$ & $5.6 \mathrm{a} \mathrm{Z}$ \\
\hline
\end{tabular}

${ }^{\mathrm{a}}$ Abbreviations: WAT, week after treatment; ${ }^{\mathrm{b}}$ Included non-ionic surfactant at $0.2 \% \mathrm{v} / \mathrm{v}$. 
At 1 WAT, UAN carrier had no significant effect on injury compared to water carrier at April 20 and May 1 application timings for the herbicides evaluated but increased injury compared to water carrier at the May 10 application timing. At 2 WAT, UAN carrier had no significant effect on injury compared to water carrier at the April 20 application timing for the herbicides evaluated but increased injury compared to water carrier at May 1 and 10 application timings (Table 3 ). When injury (1 WAT) data was separated for each herbicide, there was no difference between herbicide carriers (Water vs UAN) for bromoxynil/MCPA, dichloroprop/2,4-D, dica$\mathrm{mba} / \mathrm{MCPA} / \mathrm{mecoprop}$ or thifensulfuron/tribenuron at the April 20 or May 1 application timings. In contrast, at the May 10 application timing there was greater injury when UAN was used as the herbicide carrier with all of the herbicides tested. At $4 \mathrm{WAT}$, there was greater injury when UAN was used as the herbicide carrier for thifensulfuron/tribenuron (Table 4). UAN plus thifensulfuron/tribenuron also caused greater injury in winter wheat than UAN plus bromoxynil/MCPA, dichloroprop/2,4-D or dicamba/MCPA/mecoprop at May 10 application timing. In other studies, Lutcher and Mahler [15] found significant injury with bromoxynil plus MCPA in winter wheat when UAN was used as the carrier solution. Stahlman et al. [16] found as much as $25 \%$ injury when UAN was applied as carrier and 5\% injury when water was applied as carrier for 2,4-D, triasulfuron or 2,4-D plus triasulfuron in winter wheat at 1 WAT. In other studies when water was used as the herbicide carrier solution, Swan [19] found injury in winter wheat when 2,4-D was applied prior to tillering. Sikkema et al. [20] found as much as 7\% injury with dicamba plus MCPA plus mecoprop and minimal injury with 2,4-D amine, dichlorprop plus 2,4-D and bromoxynil plus MCPA in winter wheat. Schroeder and Banks [21] also found that earlier applications of treatments containing dicamba and dicamba plus 2,4-D, contributed to wheat injury in conventional wheat. Wicks et al. [22] and Bailey et al. [23] reported no wheat injury with thifensulfuron/tribenuronmethyl applied POST at $47 \mathrm{~g} \cdot \mathrm{ha}^{-1}$ in Virginia, USA. Hageman and Behrens [24] found as much as $11 \%$ injury with chlorsulfuron another sulfonylurea herbicide in wheat.

\subsection{Plant Height}

There was no significant reduction in winter wheat height with bromoxynil/MCPA, dichloroprop/2,4-D, dicamba/MCPA/mecoprop or thifensulfuron/tribenuron at various application timings except for dicamba/MCPA/ mecoprop treatment which reduced winter wheat height $3 \%$ at May 10 application timing compared with April 20 and May 1 application timing (Tables 1 and 2). Herbicide carrier also had no effect on winter wheat height with

Table 3. Percent visual injury and yield of winter wheat as affected by using UAN as a carrier for three herbicide application timings. Means followed by the same letter within a column (a-b) or within a row (X-Z) are not significantly different according to Fisher's Protected LSD at $\mathbf{P}<\mathbf{0 . 0 5}$.

\begin{tabular}{|c|c|c|c|c|c|}
\hline \multirow{2}{*}{ Carrier for herbicide treatment by variable ${ }^{a}$} & \multicolumn{5}{|c|}{ Application Timing } \\
\hline & Untreated & April 20 & May 1 & May 10 & SE \\
\hline & & & $\%$ & & \\
\hline \multicolumn{6}{|l|}{ Injury 1 WAT (2005) } \\
\hline Water & $0 \mathrm{a} Z$ & $0.1 \mathrm{a} Z \mathrm{ZY}$ & 0.2 a ZY & $1.0 \mathrm{a} Y$ & 0.1 \\
\hline UAN & $0 \mathrm{a} Z$ & 0.3 a Y & 0.6 a Y & $6.2 \mathrm{~b} \mathrm{X}$ & 0.4 \\
\hline SE & 0 & 0.1 & 0.2 & 0.5 & \\
\hline \multicolumn{6}{|l|}{ Injury 2 WAT } \\
\hline Water & $0 \mathrm{a} Z$ & 0.5 a Y & 0.3 a Y & 0.8 a Y & 0.1 \\
\hline UAN & $0 \mathrm{a} Z$ & 0.9 a Y & $1.0 \mathrm{~b} \mathrm{Y}$ & $3.9 \mathrm{~b} \mathrm{X}$ & 0.1 \\
\hline SE & 0 & 0.1 & 0.1 & 0.2 & \\
\hline Yield (2006 \& 2007) & & & MT $\cdot \mathrm{ha}^{-1}$ & & \\
\hline Water & 6.3 a Z & $6.4 \mathrm{a} \mathrm{Z}$ & $6.4 \mathrm{a} \mathrm{Z}$ & $6.3 \mathrm{a} \mathrm{Z}$ & 0.1 \\
\hline UAN & $6.3 \mathrm{a} \mathrm{Z}$ & $6.5 \mathrm{a} \mathrm{Z}$ & $6.3 \mathrm{a} \mathrm{Z}$ & $6.1 \mathrm{~b} \mathrm{Z}$ & 0.1 \\
\hline SE & 0 & 0.1 & 0.1 & 0.1 & \\
\hline
\end{tabular}

${ }^{\mathrm{a}}$ Abbreviations: WAT, Week after treatment; ${ }^{\mathrm{b}}$ Water and UAN were applied at $200 \mathrm{l} \cdot \mathrm{ha}^{-1}$. 
Table 4. Percent visual injury of winter wheat as affected by application timing and using UAN as a carrier for four herbicide treatments. Means followed by the same letter within a column (a-c) or row (Y-Z) for each section are not significantly different according to Fisher's Protected LSD at $\mathbf{P}<0.05$,

\begin{tabular}{|c|c|c|c|c|c|c|}
\hline \multirow{2}{*}{ Herbicide treatment by Variable } & \multicolumn{2}{|c|}{ April 20} & \multicolumn{2}{|c|}{ May 1} & \multicolumn{2}{|c|}{ May 10} \\
\hline & Water & UAN & Water & UAN & Water & UAN \\
\hline & \multicolumn{6}{|c|}{$\%$} \\
\hline \multicolumn{7}{|l|}{ Injury 1 WAT (2005) } \\
\hline Untreated & $0 \mathrm{a} Z$ & 0 a $Z$ & $0 \mathrm{a} Z$ & $0 \mathrm{a} Z$ & $0 \mathrm{a} Z$ & $0 \mathrm{a} Z$ \\
\hline Bromoxynil/MCPA & $0 \mathrm{ab} \mathrm{Z}$ & $0.2 \mathrm{~b} \mathrm{Z}$ & $0 \mathrm{ab} \mathrm{Z}$ & $0 \mathrm{ab} \mathrm{Z}$ & $0.2 \mathrm{~b} \mathrm{Z}$ & $4.9 \mathrm{bc} \mathrm{Y}$ \\
\hline Dichlorprop/2,4-D & $0.2 \mathrm{~b} \mathrm{Z}$ & $0 \mathrm{ab} \mathrm{Z}$ & $0 \mathrm{ab} \mathrm{Z}$ & $0.8 \mathrm{~b} \mathrm{Z}$ & $0.4 \mathrm{~b} \mathrm{Z}$ & $6.6 \mathrm{bc} \mathrm{Y}$ \\
\hline Dicamba/MCPA/mecoprop & $0.1 \mathrm{~b} \mathrm{Z}$ & $0.9 \mathrm{~b} \mathrm{Z}$ & $1.1 \mathrm{~b} \quad \mathrm{Z}$ & $1.7 \mathrm{~b} \mathrm{Z}$ & $1.9 \mathrm{~b} \mathrm{Z}$ & $4.2 \mathrm{~b} \mathrm{Y}$ \\
\hline Thifensulfuron/tribenuron $^{\mathrm{c}}$ & $0 \mathrm{ab} \mathrm{Z}$ & $0.4 \mathrm{~b} \mathrm{Z}$ & $0 \mathrm{ab} \mathrm{Z}$ & $0.2 \mathrm{~b} \mathrm{Z}$ & $1.8 \mathrm{~b} \mathrm{Z}$ & $9.4 \mathrm{c} \mathrm{Y}$ \\
\hline \multicolumn{7}{|l|}{ Injury 4 WAT (2006 \& 2007) } \\
\hline Untreated & $0 \mathrm{a} Z$ & 0 a Z & 0 a Z & $0 \mathrm{a} Z$ & $0 \mathrm{a} Z$ & $0 \mathrm{a} Z$ \\
\hline Bromoxynil/MCPA & $0 \mathrm{a} Z$ & 0 a Z & $0 \mathrm{a} Z$ & 0 a Z & $0 \mathrm{a} Z$ & $0 \mathrm{a} \mathrm{Z}$ \\
\hline Dichlorprop/2,4-D & $0 \mathrm{a} Z$ & $0 \mathrm{a} Z$ & 0 a $Z$ & $0 \mathrm{a} Z$ & $0 \mathrm{a} Z$ & $0 \mathrm{a} Z$ \\
\hline Dicamba/MCPA/mecoprop & 0 a Z & $0 \mathrm{a} Z$ & $0 \mathrm{a} Z$ & $0 \mathrm{a} Z$ & $0 \mathrm{a} Z$ & $0 \mathrm{a} Z$ \\
\hline Thifensulfuron/tribenuron $^{\mathrm{c}}$ & 0 a $Z$ & 0 a Z & 0 a Z & $0 \mathrm{a} Z$ & 0 a $Z$ & $2.6 \mathrm{~b} \mathrm{Y}$ \\
\hline
\end{tabular}

${ }^{\mathrm{a}}$ Abbreviations: WAT, week after treatment; UAN, urea ammonium nitrate $28 \%$ solution; ${ }^{\mathrm{b}}$ Water and UAN were applied at $2001 \cdot$ ha ${ }^{-1}$; ${ }^{\mathrm{c}}$ Included non-ionic surfactant at $0.2 \% \mathrm{v} / \mathrm{v}$.

evaluated herbicides.

In other studies when water was used as the herbicide carrier no adverse effect on winter wheat height were observed with 2,4-D amine, bromoxynil plus MCPA, or dichlorprop plus 2,4-D, however dicamba plus MCPA plus mecoprop reduced height as much as 7\% [20]. Martin et al. [25] also found 11 and $10 \%$ plant height reducetion when water was used as carrier for dicamba plus 2,4-D amine and dicamba plus MCPA in winter wheat, respectively. Wheat height reductions of $16 \%$ were also reported with dicamba, dicamba plus MCPA plus mecoprop, dicamba plus 2,4-D amine and dicamba plus MCPA when water was used as the herbicide carrier [26, 27].

\subsection{Yield}

There was no significant effect on winter wheat yield with bromoxynil/MCPA, dichloroprop/2,4-D, dicamba/ $\mathrm{MCPA} / \mathrm{mecoprop}$ or thifensulfuron/tribenuron at various application timings except for dicamba/MCPA/mecoprop treatment which reduced winter wheat yield $22 \%$ at May 10 compared to May 1 and 25\% compared to April 20 application timing in 2005 (Tables 1 and 2). UAN carrier decreased yield $3 \%$ compared to water carrier with herbicides evaluated (Table 3). In other studies when UAN was used as the carrier solution, Lutcher and Mahler [15] found significant yield reduction with bromoxynil plus MCPA in winter wheat. However, Stahlman et al. [16] found no adverse effect on yield of winter wheat when UAN or water were used as the carrier for $2,4-\mathrm{D}$, triasulfuron or 2,4-D plus triasulfuron in winter wheat. Also, when water was used as the herbicide carrier no adverse effect on winter wheat yield was observed with herbicides such as 2,4-D amine, bromoxynil plus MCPA and dichlorprop plus 2,4-D [20,27]. However, other studies have shown wheat yields were reduced as much as $39 \%$ with dicamba applied POST alone, or in combination with a phenoxy herbicide when water was used as the herbicide carrier [25,28-30]. Tottman [31] also found that tank-mixes containing dicamba, 2,3,6-TBA, MCPA or mecoprop with water as the carrier applied POST to winter wheat can reduce grain yield.

\section{Conclusion}

Results indicates that co-application of UAN and bromoxynil/MCPA, dichlorprop/2,4-D, dicamba/MCPA/mecoprop, or thifensulfuron/tribenuron may cause some initial injury in winter wheat however, crop generally recovered with no adverse effect on plant height and yield for most treatments evaluated. When there was any crop injury in winter wheat, dicamba/MCPA/mecoprop and thifensulfuron/tribenuron caused greater injury than 
bromoxynil/MCPA and dichloroprop/2,4-D (Table 1). Also, when there was any difference in application timing, the later application timing (May 10) caused greater injury than earlier application timings (April 20 or May 1). Based on this research there is potential for co-application of UAN and bromoxynil/MCPA, dichlorprop/2,4-D, dicamba/MCPA/mecoprop, or thifensulfuron/tribenuron applied postemergence in winter wheat. However, care must be taken to avoid late application timings (May 10) to reduce the chance of injury, especially with co-application of UAN with dicamba/MCPA/mecoprop and thifensulfuron/tribenuron.

\section{Acknowledgements}

The authors acknowledge Lynette Brown and Todd Cowan for their expertise and technical assistance in these studies. Funding for this project was provided in part by Grian Farmers of Ontario (GFO) and the Agricultural Adaptation Council.

\section{REFERENCES}

[1] Ontario Ministry of Agriculture and Food and Rural Affairs, "Estimated Area, Yield, Production and Farm Value of Specified Field Crops, Ontario, 2001-2008". (Available 6 August 2009).

http:/www.omafra.gov.on.ca/english/stats/crops/estimate _metric.htm

[2] B. McGee, "Estimated Area, Yield, Production and Farm Value of Specified Field Crops, Ontario, 2001-2010". (Available 8 November 2009).

http:/www.omafra.gov.on.ca/english/stats/crops/estimate _metric.htm

[3] D. R. Tottman, "Varietal Differences in the Tolerance of Cereals to Herbicides," Winter Wheat, Crop Conference, Cambridge, 17-18 December 1980, p. 68.

[4] G. J. McLeod, "Winter Cereals in Western Canada," Ecological Agriculture Projects, McGill University, Montreal, 1980, pp. 1-5.

[5] J. T. Edwards, D. B. Arnall and H. Zhang, "Nitrogen Fertilizer Timing and Source Affect Hard Red Winter Wheat Yield, but Application Method Does Not," Crop Management, 2009.

[6] J. W. Grichar and E. P. Prostko, "Effect of Glyphosate and Fungicide Combinations on Weed Control in Soybeans," Crop Protection, Vol. 28, No. 7, 2009, pp. 619-622. doi:10.1016/j.cropro.2009.03.006

[7] D. L. Jordan, A. S. Culpepper, W. J Grichar, J. TredawayDucar, B. J. Brecke and A. C. York, "Weed Control with Combinations of Selected Fungicides and Herbicides Applied Postemergence to Peanut (Arachis hypogaea L.)," Peanut Science, Vol. 30, No. 1, 2003, pp. 1-8. doi:10.3146/pnut.30.1.0001

[8] D. L. Jordan, D. Johnson and A. C. York, "Influence of Foliar Fertilizers and Pesticides on Efficacy of Selected Postemergence Herbicides," Weed Science Society of Ame- rican, Vol. 46, 2006, p. 38.

[9] S. H. Lancaster, D. L. Jordan, R. L. Brandenburg, B. Royal, B. Shew, J. Bailey, V. Curtis, A. C. York, J. W. Wilcut, J. Beam, E. Prostko, A. S. Culpepper, T. Grey, C. R. Kemerait, B. Brecke, G. McDonald, J. Tredaway-Ducar, B. College and B. Wall, "Tank Mixing Chemicals Applied to Peanut Crops: Are the Chemicals Compatible?" Extension Bulletin No. AGW653, North Carolina Cooperative Extension Service, North Carolina, 2005, p. 11.

[10] S. H. Lancaster, D. L. Jordan, J. F. Spears, A. C. York, J. W. Wilcut, D. W. Monks, R. B. Batts and R. L. Brandenburg, "Sicklepod (Senna obtusifolia) Control and Seed Production after 2,4-DB Applied Alone and with Fungicides or Insecticides," Weed Technology, Vol. 19, No. 2, 2005, pp. 451-455. doi:10.1614/WT-04-227R

[11] D. E. Robinson, N. Soltani, A. S. Hamill and P. H. Sikkema, "Weed Control in Processing Tomato (Lycopersicon esculentum) with Rimsulfuron and Thifensulfuron Applied Alone or with Chlorothalonil or Copper Pesticides," HortScience, Vol. 41, No. 5, 2006, pp. 1295-1297.

[12] M. M. Claassen and D. E. Peterson "Cheat Control in Winter Wheat with Triasulfuron and Chlorsulfuronp lus Metsulfuron," Proceedings of North Central Weed Science Society, Vol. 48, 1993, pp. 33-34.

[13] R. N. Klein and D. J. Thrailkill, "Wild Buckwheat Control in Winter Wheat with Triasulfuron," Proceedings of North Central Weed Science Society, Vol. 47, 1994, pp. 69-70.

[14] L. K. Lutcher and R. L. Mahler, "Evaluation of Urea-Ammoniumn Nitrate (UAN) Topdress Applications on Winter Wheat in Northern Idaho," Journal of Fertilizer, Vol. 2, No. 3, 1985, pp. 74-79.

[15] L. K. Lutcher and R. L. Mahler, "Sources and Timing of Spring Topdress Nitrogen on Winter Wheat in Idaho," Agronomy Journal, Vol. 80, No. 4, 1988, pp. 648-654. doi:10.2134/agronj1988.00021962008000040020x

[16] P. W. Stahlman, R. S. Currie and M. A. El-Hamid, "Nitrogen Carrier and Surfactant Increase Foliar Herbicide Injury in winter Wheat," Weed Technology, Vol. 11, 1997, pp. 7-12.

[17] G. A. Wicks, D. A. Martin and G. W. Mahnken, "Cultural Practices in Wheat (Triticum aestivum) on weeds in Subsequent Fallow and Sorghum (Sorghum bicolor)," Weed Science, Vol. 43, No. 3, 1995, pp. 434-444.

[18] G. A. Wicks and G. W. Hergert, "Effect of Fertilizer plus Herbicide Solution on Winter Wheat," Proceedings of North Central Weed Science Society, Vol. 47, 1992, pp. 29-33.

[19] D. G. Swan, "Necessity for Proper Timing of Application of 2,4-D on Winter Wheat," Down to Earth, Vol. 31, 1975, pp. 23-25.

[20] P. H. Sikkema, L. Brown, C. Shropshire and N. Soltani, "Responses of Three Types of Winter Wheat (Triticum aestivum L.) to Spring-Applied Post-Emergence Herbicides," Crop Protection, Vol. 26, No. 5, 2007, pp. 715-720. doi:10.1016/j.cropro.2006.06.010

[21] J. Schroeder and P. A. Banks, "Soft Red Winter Wheat (Triticum aestivum) Response to Dicamba and Dicamba plus 2,4-D," Weed Technology Vol. 3, No. 1, 1989, pp. 
$67-71$.

[22] G. A. Wicks, R. N. Klein, A. R. Martin and D. J. Lyon, "Annual Broadleaf Weed Control in Winter Wheat," Nebraska Cooperative Extension Report G95-1241-A, Nebraska, 1999.

[23] W. A. Bailey, H. P. Wilson, D. E. Brann and C. A. Griffey, "Wheat Cultivar Tolerance to AE F13006003," Weed Technology, Vol. 18, No. 4, 2004, pp. 881-886. doi:10.1614/WT-03-010R

[24] L. H. Hageman and R. Behrens, "Response of Small-Grain Cultivars to Chlorsulfuron," Weed Science, Vol. 29, No. 4, 1981, pp. 414-420.

[25] D. A. Martin, S. D. Miller and H. P. Alley, "Winter Wheat (Triticum aestivum) Response to Herbicides Applied at Three Growth Stages," Weed Technology, Vol. 3, No. 1, 1989, pp. 90-94.

[26] P. C. Quimby Jr. and J. D. Nalewaja, "Effect of Dicamba on Wheat and Wild Buckwheat at Various Stages of Development," Weeds, Vol. 14, No. 3, 1966, pp. 229-232. doi:10.2307/4040919

[27] N. Soltani, C. Shropshire and P. H. Sikkema, "Responses of Winter Wheat (Triticum aestivum L.) to Autumn Ap- plied Post-Emergence Herbicides," Crop Protection, Vol. 25, No. 4, 2006, pp. 346-349. doi:10.1016/j.cropro.2005.05.012

[28] J. A. Ivany, H. G. Nass and J. B. Sanderson, "Effect of Time of Application of Herbicides on Yield of Three Winter Wheat Cultivars," Canadian Journal of Plant Science, Vol. 70, No. 3, 1990, pp. 605-609. doi: $10.4141 / \operatorname{cjps} 90-076$

[29] M. J. Rinella, J. J. Kells and R. W. Ward, "Response of 'Wakefield' Winter Wheat (Triticum aestivum) to Dicamba," Weed Technology, Vol. 15, No. 3, 2001, pp. 523-529. doi:10.1614/0890-037X(2001)015[0523:ROWWWT]2.0.C O;2

[30] D. R. Tottman, "The Effects of Broad-Leaved Weed Herbicides Applied to Cereal Crops at Different Growth Stages, Aspects of Applied Biology I: Broad-Leaved Weeds and Their Control in Cereals," University of Warwick, Warwick, 1982, pp. 201-210.

[31] D. R. Tottman, "The Effects of a Dicamba Herbicide Mixture on the Grain Yield Components of Winter Wheat," Weed Research, Vol. 18, No. 6, 1978, 335-339. doi:10.1111/j.1365-3180.1978.tb01170.x 